\title{
propriétés géotechniques des tuffs volcaniques du projet hydroélectrique Larreynaga au Nicaragua
}

\author{
geotechnical properties of volcanic tuffs \\ for Larreynaga hydroelectric project, Nicaragua
}

\author{
J. BINQUET \\ Ingénieur en Chef \\ COYNE et BELLIER (*) \\ Bureau d'Ingénieurs-Conseils
}

Rev. Franç, Géotech. n 47, p.p. $61-67$ (avril 1989)

\section{Résumé}

Le barrage de $40 \mathrm{~m}$ de hauteur qui fait partie du projet hydroélectrique Larreynaga sera fondé sur un substratum constitué de laves (en fond de vallée) et de tuffs volcaniques sur les rives. Les laves sont soit des basaltes soit des andésites et possèdent des propriétés mécaniques qui sont celles des roches. Les tuffs qui résultent du dépôt et de l'accumulation de cendres, de lapillis et bombes volcaniques projetées dans l'atmosphère sont des matériaux très hétérogènes (par la taille des grains, par la nature des minéraux présents, par la cimentation et par le degré d'altération) qui s'apparentent à des sols. L'étude du barrage a donc conduit le projeteur à une étude détaillée des propriétés géotechniques particulières de ces sols. Ce sont les principaux résultats de cette étude qui sont exposés dans le présent article.

Outre ces caractéristiques, la communication présente le contexte géologique dans lequel se trouve placé le projet étudié et met l'accent sur l'analyse qui a été faite de ces propriétés géotechniques pour en tirer les paramètres de conception du projet du barrage.

\section{Abstract}

The $40 \mathrm{~m}$ high dam in the Larreynaga hydro-project will be built on lava, at the bottom of the valley, and volcanic tuffs in the abutments. The lava is composed of basalts and andesites with the mechanical properties of rock, whereas the tuffs, which are the build-up of ash, lapilii, and volcanic bombs, are a very heterogeneous mixture (in terms of grain sizes, minerals, cementation, and weathering) closer to soils in nature. Designing the dam thus required detailed investigation of the specific geotechnical properties of these soils, the main results of which are presented hereafter.

In addition, the paper outlines the project's geological context and underscores the analysis of the geotechnical properties leading to the basic design parameters for the dam. 


\section{PRÉAMBULE}

Les études de conception définitive du projet hydroélectrique Larreynaga ont permis de mettre en évidence des propriétés géotechniques particulières des tuffs volcaniques constituant l'essentiel des fondations des ouvrages de ce projet. Ce projet, situé à proximité de la ville de Jinotega (voir fig. 1 ci-dessous) comprend un barrage en remblais de $40 \mathrm{~m}$ de hauteur et ses ouvrages annexes, une prise d'eau, un canal de $3300 \mathrm{~m}$ de long, une usine hydroélectrique de $41 \mathrm{MW}$ de puissance installée et des ouvrages de fuite de $600 \mathrm{~m}$ de longueur. Les travaux de reconnaissances géologiques et géotechniques nécessaires aux études du projet ont permis de distinguer deux grands types de roches sur le site:

- les laves qui sont les seules qui méritent vraiment le nom de roches: elles possèdent une excellente dureté et ne se délitent pas dans l'eau;

- les tuffs qui, selon les cas, peuvent être considérés comme des roches tendres ou des sols compacts. Tous les échantillons, même les plus indurés, sont sensibles à l'eau et se délitent plus ou moins rapidement par immersion.

Ces formations appartiennent aux groupes de roches volcaniques Coyol et Matagalpa et sont d'âge tertiaire (Miocène moyen). Les laves proviennent de la solidification d'un magma liquide sortant d'un cratère volcanique tandis que les tuffs résultent de l'accumulation d'éléments de toutes dimensions : cendres, lapillis et bombes volcaniques projetés dans l'atmosphère lors des éruptions volcaniques. Le présent article ne traite que des caractéristiques géotechniques des tuffs.

La zone du projet se trouve à une altitude comprise entre 800 et $100 \mathrm{~m}$ au-dessus du niveau de la mer et est caractérisée par les principales données climatologiques suivantes qui sont celles d'un climat tropical pluvieux :

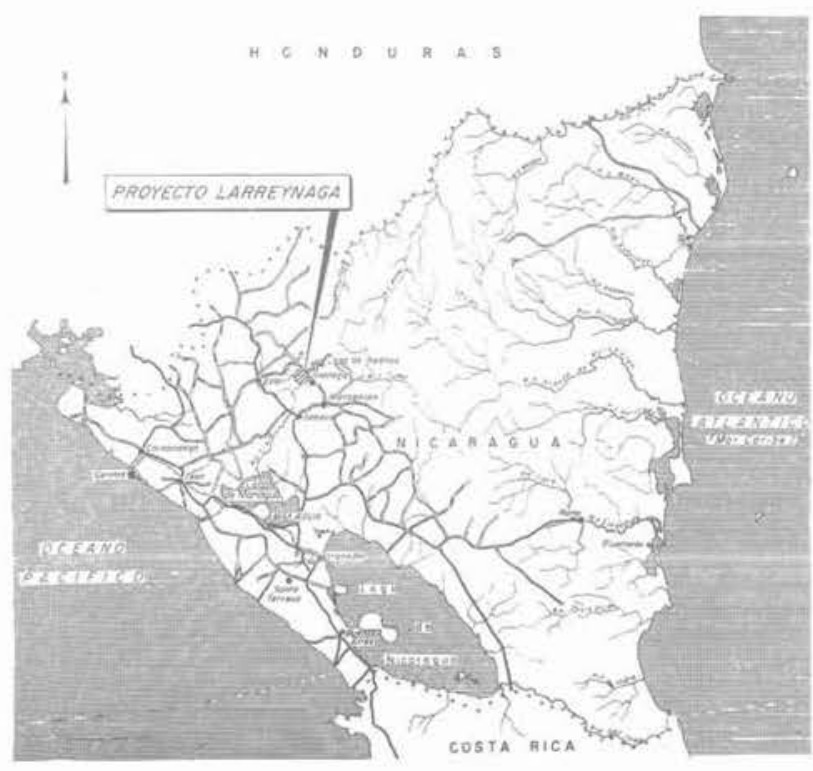

Fig. 1. - Carte du Nicaragua.

Fig 1, - Map of Nicaragua
- les températures moyennes mensuelles varient peu tout au long de l'année puisqu'elles sont comprises entre $18.5^{\circ} \mathrm{C}$ (mois de janvier) et $21.4^{\circ} \mathrm{C}$ (mois de mai) ;

- les valeurs moyennes annuelles de l'humidité relative sont généralement comprises entre 75 et $95 \%$, les valeurs moyennes mensuelles étant, elles, comprises entre $80 \%$ (avril) et $89 \%$ (juillet) ;

- la valeur moyenne annuelle de la pluviométrie est de l'ordre de $1450 \mathrm{~mm}$ avec des moyennes mensuelles comprises entre $18 \mathrm{~mm}$ (mars) et $228 \mathrm{~mm}$ (juin).

\section{PRÉSENTATION GÉNÉRALE}

Ces tuffs se présentent sous des formes extrêmement variées suivant les caractéristiques granulométriques de ses éléments constituants. On peut distinguer en effet:

- les tuffs fins, constitués presque exclusivement de cendres fines de couleur blanche à rosée ;

- les tuffs sableux formés par des éléments de la taille de sables moyens et grossiers cimentés par des cendres fines, de couleur beige à grise ;

- les tuffs « aglomératiques „ qui se caractérisent par la présence de lapillis et de bombes volcaniques de toutes dimensions (jusqu'à $0,5 \mathrm{~m}$ de diamètre) également cimentés par les cendres fines ;

- les paléosols qui se trouvent en général à la base des coulées de lave et qui se présentent comme de la brique rouge. Il s'agit d'argile, qui a été portée à haute température par le magma :

- les brèches et conglomérats qui sont des éléments de transition entre les laves et les tuffs et se trouvent généralement dans la partie supérieure des coulées de lave.

\section{CARACTÉRISTIQUES D'IDENTIFICATION}

Compte tenu des variations des caractéristiques d'identification des tuffs en fonction de la position en profondeur des échantillons prélevés, les tuffs de surface (profondeur inférieure à $5 \mathrm{~m}$ ) ont été distingués des tuff's profonds (entre $5 \mathrm{~m}$ et $36 \mathrm{~m}$ de profondeur).

\subsection{Caractéristiques des tuffs de surface}

Ces matériaux sont des matériaux fins ou sablograveleux dont la fraction fine est peu ou moyennement plastique. Ces matériaux peuvent être classés ML. MH, SM ou GM dans la classification de IU.S.C.S.

Le diamètre maximum des grains est compris entre 5 et plus de $100 \mathrm{~mm}$, le pourcentage d'éléments inférieurs à 80 microns est compris entre 4 et 67 (26\% en moyenne sur 30 échantillons), la limite de liquidité est comprise entre 33 et $67 \%$ (49\% en moyenne), l'indice de plasticité est compris entre 5 et $29 \%$ (15\% en moyenne) et la densité spécifique des grains est comprise entre 2,31 et 2,71 (2,62 en moyenne). Ces matériaux se trouvent à une teneur 
en eau à l'état naturel qui est comprise entre 8 et $35 \%$ (19\% en moyenne). La comparaison entre les limites d'ATTERBERG obtenues avec celles citées par MITCHELL et SITAR (1982), montre que les tuffs de surface appartiennent à la zone commune des deux catégories d'Oxysols et d'Andosols. La figure 2 présente le diagramme de plasticité de ces matériaux.

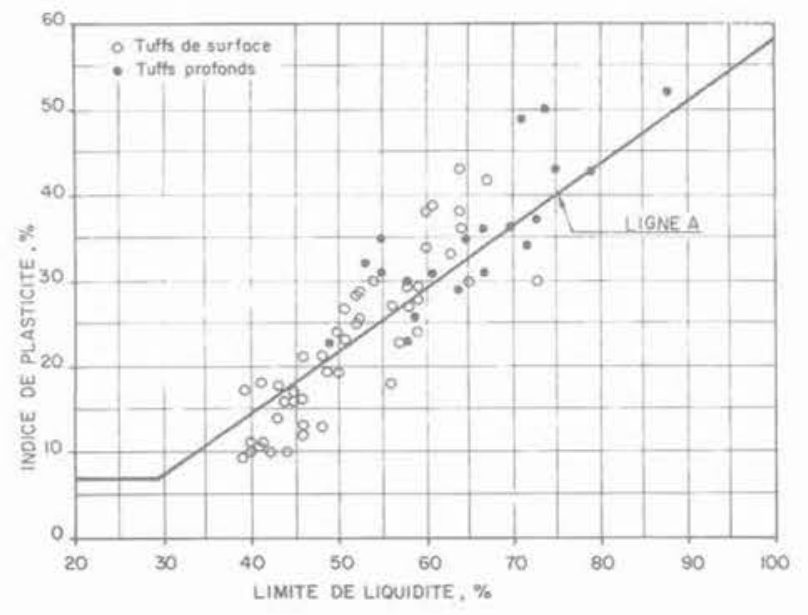

Fig. 2. - Diagramme de Casagrande.

Fig. 2. - Casagrande Classification Chart.

\subsection{Caractéristiques des tuffs profonds}

Ces matériaux sont essentiellement des matériaux sablo-limoneux qui comprennent une fraction argileuse réduite bien que relativement plastique. Ces matériaux peuvent être classés SM, SC, SP-SC et SP-SM dans la classification de IU.S.C.S.

Leur pourcentage d'éléments inférieurs à 80 microns est compris entre 7 et $45 \%$ (18\% en moyenne sur 20 échantillons), la limite de liquidité est comprise entre 49 et $88 \%(66 \%$ en moyenne), l'indice de plasticité est compris entre 23 et $52 \%$ (35\% en moyenne) et la densité spécifique des grains est de l'ordre de 2,70. Ces matériaux se trouvent à une teneur en eau à l'état naturel qui est comprise entre 5 et $35 \%$ (22\% en moyenne) avec des degrés de saturation compris entre $90 \%$ et $100 \%$. La figure 2 présente le diagramme de plasticité de ces matériaux. Il faut préciser que les teneurs en eau mentionnées (limites et teneur en eau à l'état naturel) ont été obtenues après étuvage à une température de $105^{\circ}$ durant 24 heures. Pour un certain nombre d'échantillons, on a également effectué des mesures de teneur en eau à une température de $60^{\circ}$ durant 72 heures. La comparaison de ces résultats montre que les teneurs en eau mesurées à $105^{\circ}$ sont supérieures à celles mesurées à $60^{\circ}$ et que la différence relative de teneur en eau est comprise entre 10 et $31 \%$ ( $20 \%$ en moyenne). Par ailleurs la comparaison entre les limites d'ATTERBERG de ces tuffs et les valeurs définies par MITCHELL et SITAR (1982) montre que les matériaux appartiennent au groupe des Oxysols ce qui est confirmé par les résultats des analyses chimiques présentées ci-après, Il faut ajouter que, comme l'a souligné TOWNSEND (1985), la détermination des limites d'ATTERBERG de ces maté- riaux est très sensible au séchage et au remaniement. Le séchage tend à diminuer la plasticité tandis que le remaniement peut entraîner l'effet inverse. Un des effets du séchage est d'augmenter la cimentation par oxydation des sesquioxydes de fer et d'aluminium. Quant au remaniement, son effet est de rompre les relations de cimentation qui existent entre les oxydes de fer ou d'aluminium et les agrégats d'argile.

\section{CARACTÉRISTIQUES DE COMPACITÉ}

Comme on le verra ci-après, le paramètre le plus important qui régit les propriétés mécaniques de ces tuffs est la compacité exprimée soit sous la forme de l'indice des vides $e$, soit sous la forme du poids volumique sec, $\gamma_{\mathrm{d}}$. Les mesures du poids volumique sec qui ont été effectuées (56 mesures au total entre 3 et $36 \mathrm{~m}$ de profondeur) montrent la forte augmentation de la compacité avec la profondeur puisque l'indice des vides moyen passe de 0,8 (avec un écart type de 0,16 ) en surface à près de 0,6 (écart type de 0,18 ) en profondeur. L'ensemble de ces résultats a permis d'élaborer un profil type de variation de l'indice des vides avec la profondeur. Ce profil est indiqué dans le tableau 1 ci-dessous.

Tableau 1. Profil-type de compacité

\begin{tabular}{|r|c|c|c|c|}
\hline Prof., m & $\begin{array}{c}\mathrm{e} \\
\mathrm{moy}\end{array}$ & $\begin{array}{c}\mathrm{e}^{*} \\
\max .\end{array}$ & Gs & $\begin{array}{c}\gamma_{d} \mathrm{moy.} \\
\mathrm{kN} / \mathrm{m}^{3}\end{array}$ \\
\hline 3,0 à 5,0 & 0,80 & 0,88 & 2,62 & 14,6 \\
5,0 à 12,0 & 0,70 & 0,78 & 2,66 & 15,6 \\
12,0 à 36,0 & 0,60 & 0,69 & 2,70 & 16,90 \\
\hline
\end{tabular}

e max $=$ e moy. $+50 \%$ de lécart type.

\section{CARACTÉRISTIQUES MÉCANIQUES}

On distingue ci-après les caractéristiques mécaniques à court terme obtenues par des essais de cisaillement rapides non consolidés non drainés et celles à long terme obtenues à l'aide d'essais de cisaillement lents consolidés drainés ou rapides consolidés non drainés mais avec mesure de pression interstitielle. Les matériaux soumis aux essais sont des tuffs prélevés en surface ou en profondeur de compacité très variable.

\subsection{Caractéristiques mécaniques à court terme}

Les valeurs des résistances à la compression simple obtenues varient considérablement d'un point à un autre dans une même zone. Ces valeurs sont très éle- 
vées pour des sols même de grande compacité et apparentent ces matériaux à des roches tendres. Le paramètre qui paraît le plus important pour expliquer ces grandes variations de résistance à la compression simple est la compacité. La figure 3 présente les valeurs de la résistance obtenue en fonction du poids volumique sec. Cette figure montre la tendance bien marquée de laugmentation de la résistance avec le poids volumique.

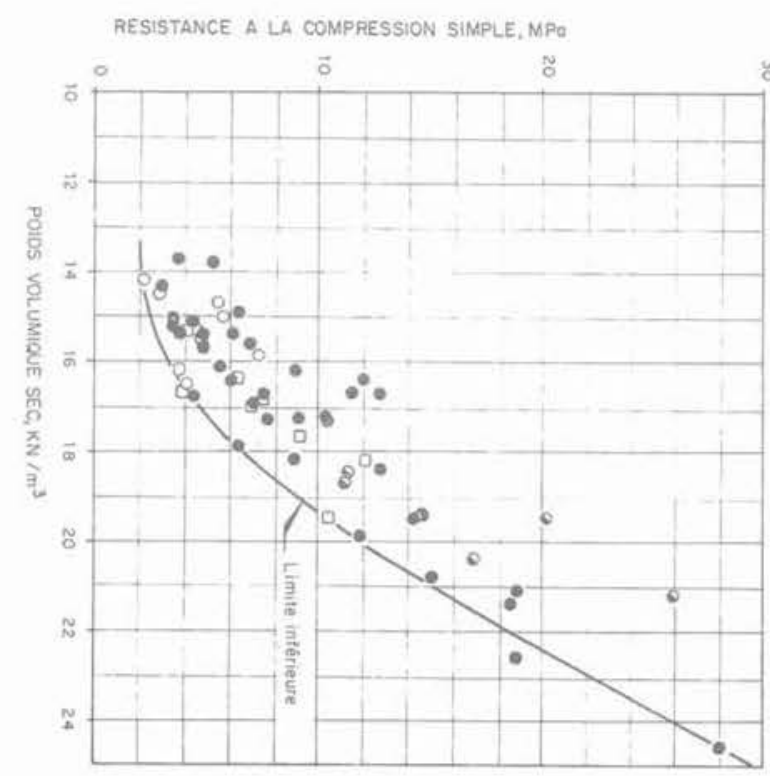

Fig. 3. - Relation entre résistance á la compression et compacité

Fig 3. - Relationship between undrained shear strength and density.

\subsection{Caractéristiques mécaniques à long terme}

Les caractéristiques mécaniques à long terme ont été obtenues à l'aide d'essais de cisaillement lent lessais $C D$ triaxiaux avec une vitesse de cisaillement contrôlée de $1 \mathrm{micron} / \mathrm{min}$. ou à la boite de Casagrande avec une vitesse de cisaillement de 6,7 microns/min.) ou d'essais triaxiaux rapides avec mesure de pression interstitielle sur éprouvettes consolidées et non drainées (vitesse de cisaillement de 40 microns/min.). L'interprétation de certains essais s'est révélée difficile surtout lorsque le comportement des matériaux ressemblait davantage à celui d'une roche que celui d'un sol. En effet, pour ces matériaux, la courbe intrinsèque possède une forme parabolique bien marquée. caractéristique des échantillons rocheux. Si les essais triaxiaux lents $C D$ ont pu être convenablement interprétés, les essais triaxiaux $\mathrm{CU}$ ont conduit, semble-til, à surestimer les caractéristiques mécaniques effectives car compte tenu de la grande rigidité de certaines éprouvettes (due à la surconsolidation et à la cimentation) et à leur comportement fortement dilatant, les pressions interstitielles mesurées à l'extrémité de l'échantillon doivent sous-estimer considérablement les pressions existantes sur les surfaces de rupture. Aussi a-t-on considéré avec beaucoup de prudence certains des résultats obtenus par ces essais triaxiaux $\mathrm{CU}$.
Les résultats de ces essais et leur interprétation en termes de résistance de pic (déviateur maximum) fournissent des valeurs très dispersées tant pour l'angle de frottement interne (variable entre 23 et plus de $60^{\circ}$ ) que pour les cohésions effective ou drainée (variable entre 83 et plus de $1000 \mathrm{kPa}$ ). On a donc effectué deux tentatives de corrélation de l'angle de frottement interne, d'une part avec l'indice de plasticité, sans succès, et d'autre part avec l'indice des vides initial des échantillons. La figure 4 présente cette seconde corrélation qui peut être estimée convenable si on exclut certains résultats tirés des essais triaxiaux $\mathrm{CU}$. Cette corrélation ainsi qu'une corrélation analogue tentée avec la cohésion effective (entre la cohésion et l'indice des vides) ont permis de déduire les caractéristiques minimales de cisaillement (résistance de pic) de ces tuffs volcaniques comme indiqué dans le tableau II.

Tableau II. Caractéristiques mécaniques minimales (Résistance de pic)

\begin{tabular}{|c|c|c|}
\hline $\begin{array}{c}\text { Indice des vides } \\
\mathrm{e}\end{array}$ & $\begin{array}{c}\text { Angle } \\
\text { de frottement } \\
\phi \text { ou } \phi d, d^{\circ}\end{array}$ & 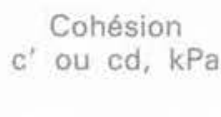 \\
\hline $\begin{array}{l}0,80 \\
0,70 \\
0,60\end{array}$ & $\begin{array}{l}26,5 \\
29,5 \\
34,5\end{array}$ & $\begin{array}{r}70 \\
85 \\
150\end{array}$ \\
\hline
\end{tabular}

A propos de ces caractéristiques mécaniques de pic, il est important de faire les remarques suivantes:

- les fortes cohésions obtenues révèlent la nature rocheuses de ces matériaux (forte surconsolidation et forte cimentation qui est due en partie aux fortes teneurs d'oxydes ferriques et d'aluminium) puisque les essais ont été réalisés avec des contraintes de consolidation inférieures aux contraintes de préconsolidation et que les opérations de saturation-consolidation des échantillons n'ont pu détruire la cimentation. Pour examiner comment peut être détruite cette cimentation, on a réalisé des essais de délitage au laboratoire. Ces essais ont montré que le délitage a été obtenu sur des échantillons non confinés après un ou plusieurs cycles (jusqu'à 5) de saturation-séchage à l'air à $40^{\circ} \mathrm{C}$. Il faut souligner que ce type d'essais est beaucoup plus sévère que le "quantitative slaking test" décrit par MORGENSTERN et EIGENBROD (1974) ;

- la forte cimentation et surconsolidation confèrent aux échantillons un comportement très rigide (déformation à la rupture relativement faible comprise entre 1 et $2,5 \%$ ) et fragile (forte perte de résistance après le pic) ;

- la forte surconsolidation confère un comportement très dilatant durant le cisaillement attesté d'une part par les variation de volume $(\Delta \mathrm{V} / \mathrm{V})$ dans les essais drainés et d'autre part par les coefficients de pression interstitielle à la rupture (Af) dans les essais non drainés. Ainsi les valeurs de $\Delta V / V$ à la rupture sont toujours positives ( $1 \%$ en moyenne) et les valeurs de Af sont voisines de $0(0,01$ à 0,06$)$.

Ces observations montrent que les caractéristiques mécaniques de pic ne peuvent être utilisées dans une éude de stabilité de remblais fondés sur ces tuffs 


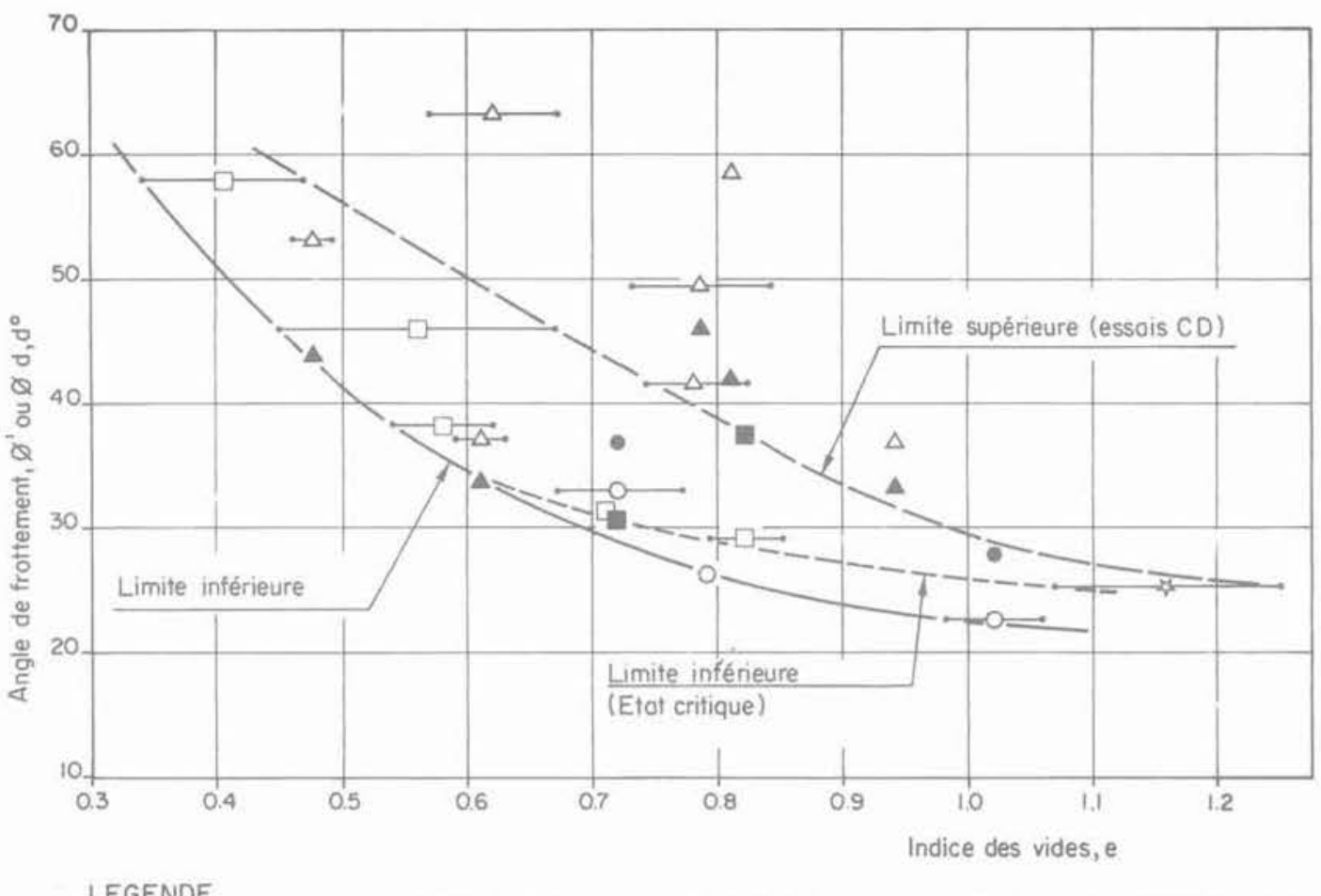

TRIAXIAL CD

TRIAXIAL CU

BOITE DE CISAILLEMENT

Résistance de pic
Rèsistance de l'état
critique

$\Delta$

0 critique

Fig. 4. - Relation entre l'angle de frottement drainé et l'indice des vides.

Fig. 4. - Relationship between drained or effective friction angle and void ratio.

puisqu'il n'existe pas d'homogénéité de comportement entre les remblais peu rigides (déformation à la rupture supérieure à $4 \%$ environ pour les argiles du noyau du barrage) et peu fragiles (faible perte de résistance après le pic). Ces différences de comportement font penser que les matériaux rigides agissent comme concentrateurs de contraintes qui peuvent ainsi initier une rupture progressive. Ce type de rupture a été étudiée par SKEMPTON (1964) et d'autres pour les fondations d'argiles surconsolidées fissurées. Pour ces argiles, SKEMPTON $(1970,1977)$ a montré que la résistance de ces argiles est la résitance de pic du matériau normalement consolidé, laquelle se produit juste avant que l'état critique tel que défini par $\mathrm{SCHO}$ FIELD et WROTH (1968) soit atteint, cet état critique est tel que toute augmentation de la contrainte de cisaillement n'induit pas de changement de teneur en eau. Bien que le problème des tuffs soit différent de celui des argiles surconsolidées fissurées, le risque de destruction de la cimentation, la fragilité et la dilatance de ces matériaux font penser que l'état critique est une bonne approche de la résistance de ces matériaux quand on peut craindre une rupture progressive. Les essais mécaniques présentés précédemment ont donc été interprétés en termes d'état critique.

Les points correspondants à langle de frottement interne ont reportés sur la figure 4 précitée. Ces résul- tats ont alors permis de déduire les caractéristiques minimales de cisaillement à l'état critique applicables à ces matériaux (voir tableau III).

Tableau III. Caractéristiques mécaniques minimales (Résistance à l'état critique)

\begin{tabular}{|c|c|c|}
\hline $\begin{array}{c}\text { Indice des vides } \\
e\end{array}$ & $\begin{array}{c}\text { Angle } \\
\text { de frottement } \\
\phi^{\prime} \text { ou } \phi \mathrm{d}, \mathrm{d}^{\circ}\end{array}$ & $\begin{array}{c}\text { Cohésion } \\
\mathrm{C}^{\prime} \text { ou Cd, kPa }\end{array}$ \\
\hline 0,80 & 29,0 & 0 \\
0,70 & 31,0 & 0 \\
0,60 & 34,5 & 0 \\
\hline
\end{tabular}

\section{CARACTÉRISTIQUES DE GONFLEMENT}

Des essais cedométriques ont été réalisés tant sur les tuffs de surface que sur les tuffs profonds afin d'apprécier leurs caractéristiques de gonflement. 
Ces essais ont consisté à saturer les échantillons après application d'un chargement équivalent à la contrainte effective verticale in situ puis à poursuivre l'essai jusqu'à atteindre une charge de $5 \mathrm{MPa}$. les gonflements observés sont généralement faibles, compris entre 0,3 et $1,2 \%$. Les indices de gonflement sont compris entre 0.01 et 0.04 et l'indice de compression est de l'ordre de 0,13. Les pressions de préconsolidation constatées sont élevées et dépassent généralement 1,5 à $2 \mathrm{MPa}$, ce qui donne des rapports de surconsolidation compris entre 5 et 10 . Pour ces valeurs du rapport de surconsolidation, le coefficient des terres au repos, Ko, évalué par la formule de MAYNE et KULHAWY (1982) est compris entre 1,1 et 1,5 .

\section{CARACTÉRISTIQUES MINÉRALOGIQUES ET CHIMIQUES}

Les analyses minéralogiques et chimiques effectuées sur des échantillons de tuffs (lames minces colorées, diffraction de rayons $X$ et analyses chimiques) montrent que les minéraux les plus fréquents sont des Plagioclases (de type Labradorite), des Pyroxènes (Augites), des Clinoptilotites (Heulandite ou Zeolite), des Magnétites et des Biotites, ce qui d'après MOHR et al (1972) caractérise un matériau de la famille des Andésites Dioritiques. L'analyse de la fraction argileuse met en évidence la présence de Montmorillonite (Bentonite) en petite quantité. Compte tenu de la faible fraction argileuse de ces échantillons (entre 0 et $8 \%$ d'éléments inférieurs à 2 microns), la présence de minéraux très actifs est confirmé par les indices de plasticité relativement élevés ou plus exactement par des valeurs d'activité très élevées (entre 4,0 et 9,0 ). II faut signaler, en outre, que la présence de Zéolite est la caractéristique d'une action fumerolienne importante et d'une activité hydrothermale. Du point de vue chimique, il faut noter la forte proportion des oxydes de fer et d'aluminium ( 25 à $38 \%$ ) ce qui permet de classer ces matériaux dans les groupes des Latosols ou Oxysols d'après MITCHELL (1976) mais ne permet pas de les inclure dans les familles des sols latéritiques (rapport $\mathrm{SiO}_{2} / \mathrm{Al}_{2} \mathrm{O}_{3}$ inférieur à 2,0 ) d'après BAWA (1957). Notons également que l'absence de minéraux amorphes (allophane) et d'halloysite les distingue des sols allophaniques ou Andosols.

\section{CARACTÉRISTIQUES DE DISPERSIVITÉ}

On a réalisé sur ces matériaux deux types d'essais essais du trou d'aiguille (pinhole test) d'après la méthode développée par SHERARD et al (janvier 1976 et avril 1976) sur échantillons intacts et analyses chimiques de l'extrait de saturation selon la méthode développée par SHERARD et al (1972).

Les essais du trou d'aiguille montrent que ces tuffs sont une roche tendre non dispersive (ND2) tandis que les analyses chimiques classent les échantillons dans la zone A (sols dispersifs). CRAFT et ACCIARDI (1984) ont montré récemment que ce type d'analyse chimique n'avait pas réussi, dans de nombreux cas, à identifier convenablement des sols dispersifs. Bien que SHERARD (1985) mette en doute ces conclusions, il admet qu'il existe des catégories de sols pour lesquels les analyses chimiques ne confirment pas les essais physiques et notamment lorsque le sol contient principalement de la kaolinite ou des oxydes de fer ou d'autres minéraux qui contrarient les effets du sodium. D'après MEDINA et al (1982) un tel phénomène a été observé sur les sols de barrage de GURI au Vénézuela. Pour SHERARD, l'essai du trou d'aiguille reste, pour le moment, l'essai le plus fiable pour détecter les matériaux dispersifs.

\section{CONCLUSIONS}

Les tuffs volcaniques de la fondation du projet Larreynaga possèdent des propriétés géotechniques particulières :

- la fraction argileuse est faible mais très active :

- les propriétés mécaniques à court terme mais aussi à long terme sont essentiellement commandées par l'indice des vides:

- la forte cimentation et surconsolidation confèrent un comportement rigide et très dilatant durant le cisaillement, ce qui fait penser que la résistance à l'état critique est une bonne approche de la résistance de ces matériaux quand on peut craindre une rupture progressive ;

- les analyses chimiques classent ces matériaux dans la catégorie des sols dispersifs tandis que les essais du trou d'aiguille montrent que ces matériaux sont non-dispersifs, la forte teneur en oxyde de fer pouvant peut-être expliquer cette contradiction :

- les caractéristiques d'identification comme les résultats des analyses chimiques permettent de classer ces matériaux dans la famille des Latosols ou Oxysols.

\section{BIBLIOGRAPHIE}

1. BAWA K.S. (1957), Laterite Soils and their Engineering Characteristics. Journal of the Soils Mechanics and Foundation Engineering Division, ASCE, vol. 82, 1976, N SM4.

2. MAYNE P., KULHAWY F.H. (1982), Ko-OCR Relationships in Soil. Proc. ASCE, Journal of Geotechnical Engineering Division, vol. 108. No. GT6, June

3. CRAFT D.C., ACCIARDI R.G. (1984), Failure of Pore-Water Analyses for Dispersion, Journal of Geotechnical Engineering, vol. 110, $\mathrm{N}^{\circ} 4$, pp. 459-472.

4. MEDINA J. et al (1982), Chemical and Mineralogical Evaluation of Foundation Soils at Guri Dam, Venezuela. Proc. 14th ICOLD, vol. II Rio de Janeiro, Brazil, pp. 261-274. 
5. MITCHELL J.K., SITAR N. (1982), Engineering Properties of Tropical Residual Soils. ASCE Geotechnical Engineering Specialty Conference on Engineering and Construction in Tropical and Residual Soils, Honolulu, Hawaï.

6. MITCHELL J.K. (1976), Fundamentals of Soils Behavior. John Wiley and Sons, Inc., New York.

7. MOHR E.C., VAN BOREN F.A., VAN SCHUYLENBORGH J. (1972), Tropical Soils. 3rd Edition, Mouon-Ichtior-Van Houve, The Hague, The Netherlands.

8. MORIN W.J., TODOR P.C. (1975), Laterite and Lateritic Soils and other Problem Soils of the Tropics. USAID 3682, Lyon Associates, Baltimore.

9. ROSCOE K.H., SCHOFIELD A.N., WROTH C.P. (1958), On the yielding of soils. Geotechnique, vol. $8, \mathrm{~N}^{\circ} 1, \mathrm{pp} .22-53$.

10. SCHOFIELD A.N., WROTH C.P. (1968), Critical State Soils Mechanics. Mc Graw Hill, London.

11. SHERARD J.L., DUNNIGAN L.P., DECKER R.S., STEEL E.F. (1976), Pinhole Test for identifying Dispersive Soils. Journal of Geotechnical Engineering Division, vol. 102, N GT1. pp. 69-85.
12. SHERARD J.L., DUNNIGAN L.P., DECKER R.S. (1976), Identification and nature of Dispersive Soils. Journal of Geotechnical Engineering Division, vol. 102, No GT4, pp. 287-301.

13. SHERARD J.L., DECKER R.S., RYKER N.L. (1972), Piping in Earth Dams of Dispersive Clay, Proc. of the ASCE Speciality Conference on the performance of Earth and Earth-Supported Structures, Purdue University.

14. SHERARD J.L. (1985), Discussion of the paper by CRAFT and ACCIARDI. Journal of Geotechnical Engineering, vol. 111, No 8 , pp. 1050-1052.

15. SKEMPTON A.W. (1964), Long term stability of clay slopes. Geotechnique, vol. 14, pp. 71-102.

16. SKEMTPON A.W. (1970), First-time slides in overconsolidated clays. Geotechnique, vol. 20, No 3, pp. 77-102.

17. SKEMPTON A.W. (1977), Slope Stability of Cuttings in Brown London Clay. Proc. of the IX International Conference on Soil Mechanics and Foundation Engineering, Tokyo, vol. 3. pp. 261-270

18. TOWNSEND F.C. (1985), Geotechnical Characteristics of Residual Soils. Proc. ASCE, Journal of Geotechnical Engineering, vol. 111, $\mathrm{N}^{0} 1$. 